\title{
Quantum Algorithm for Full Configuration Interaction Calculations without Controlled Time Evolutions
}

Kenji Sugisaki, Chikako Sakai, Kazuo Toyota, Kazunobu Sato, Daisuke Shiomi and Takeji Takui

\begin{tabular}{|c|l|}
\hline Citation & The Journal of Physical Chemistry Letters. 12(45); 11085-11089 \\
\hline Issue Date & $2021-11-18$ \\
\hline Type & Journal Article \\
\hline Textversion & Publisher \\
\hline $\begin{array}{c}\text { Supporting } \\
\text { Information }\end{array}$ & $\begin{array}{l}\text { The Supporting Information is available free of charge at } \\
\text { https://pubs.acs.org/doi/10.1021/acs.jpclett.1c03214. }\end{array}$ \\
\hline Rights & $\begin{array}{l}\text { C } 2021 \text { The Authors. Published by American Chemical Society. This work is } \\
\text { licensed under a Creative Commons Attribution-NonCommercial-NoDerivatives } \\
\text { 4.0 International License. https://creativecommons.org/licenses/by-nc-nd/4.0/ }\end{array}$ \\
\hline DOI & $10.1021 /$ acs.jpclett.1c03214 \\
\hline
\end{tabular}

\author{
Self-Archiving by Author(s) \\ Placed on: Osaka City University Repository
}

Sugisaki, K., Sakai, C., Toyota, K., Sato, K., Shiomi, D., \& Takui, T. (2021). Quantum Algorithm for Full Configuration Interaction Calculations without Controlled Time Evolutions. The Journal of Physical Chemistry Letters. 12, 11085-11089. https://doi.org/10.1021/acs.jpclett.1c03214 


\begin{tabular}{|c|c|}
\hline Highlights & $\begin{array}{l}\text { ○量子コンピュータを用いれば、精密な量子化学計算を分子サイズに対して従来の } \\
\text { コンピュータよりも指数関数的に少ない計算ステップで実行できることが理論的に } \\
\text { 示されている。 } \\
\diamond \text { 量子コンピュータでの精密量子化学計算手法である量子位相推定は、量子ゲート } \\
\text { の並列処理がしにくい、隣り合わない } 2 \text { 量子ビットにかかる量子ゲートが多いなど } \\
\text { の問題を抱えている。 } \\
\diamond \text { 先行開発した量子位相差推定アルゴリズムを応用し、従来法よりも量子コンピュ } \\
\text { ータ実機に実装しやすい新規量子化学計算量子アルゴリズムを開発。 }\end{array}$ \\
\hline Description & $\begin{array}{l}\text { 【概要】 } \\
\text { 研究チームは、原子・分子の全エネルギーを計算する新規量子アルゴリズムを開 } \\
\text { 発しました。今回開発した量子アルゴリズムは従来法よりも量子ゲートの並列実行 } \\
\text { 性に優れ、量子コンピュータ実機への実装が格段に容易になることが期待されます。 } \\
\text { 【今後の展開と応用】 } \\
\text { 今回開発した量子位相差推定による全エネルギー計算手法は量子ゲートの並列処 } \\
\text { 理がしやすくなるだけでなく、物理的に隣り合わない } 2 \text { つの量子ビットに作用する } 2 \\
\text { 量子ビットゲートの数も劇的に減らすことができ、従来法よりも量子コンピュータ } \\
\text { 実機への実装が容易になります。将来、本手法が量子コンピュータによる精密量子 } \\
\text { 化学計算の代名詞となるかもしれません。 } \\
\text { ‘高精度量子化学計算の新手法！量子コンピュータで原子・分子の全エネルギーを計 } \\
\text { 算する新しい手法を提案 ! ’. 大阪市立大学. } \\
\text { https://www.osaka-cu.ac.jp/ja/news/2021/211112-1 (参照 2021-11-12) }\end{array}$ \\
\hline
\end{tabular}

Sugisaki, K., Sakai, C., Toyota, K., Sato, K., Shiomi, D., \& Takui, T. (2021). Quantum Algorithm for Full Configuration Interaction Calculations without Controlled Time Evolutions. The Journal of Physical Chemistry Letters. 12, 11085-11089. https://doi.org/10.1021/acs.jpclett.1c03214 


\title{
Quantum Algorithm for Full Configuration Interaction Calculations without Controlled Time Evolutions
}

\author{
Kenji Sugisaki,* Chikako Sakai, Kazuo Toyota, Kazunobu Sato,* Daisuke Shiomi, and Takeji Takui* \\ Cite This: J. Phys. Chem. Lett. 2021, 12, 11085-11089 \\ Read Online
}

ABSTRACT: A quantum phase estimation algorithm allows us to perform full configuration interaction (full-CI) calculations on quantum computers with polynomial costs against the system size under study, but it requires quantum simulation of the time evolution of the wave function conditional on an ancillary qubit, which makes the algorithm implementation on real quantum devices difficult. Here, we discuss an application of the Bayesian phase difference estimation algorithm that is free from controlled time evolution operations to the full-CI calculations.

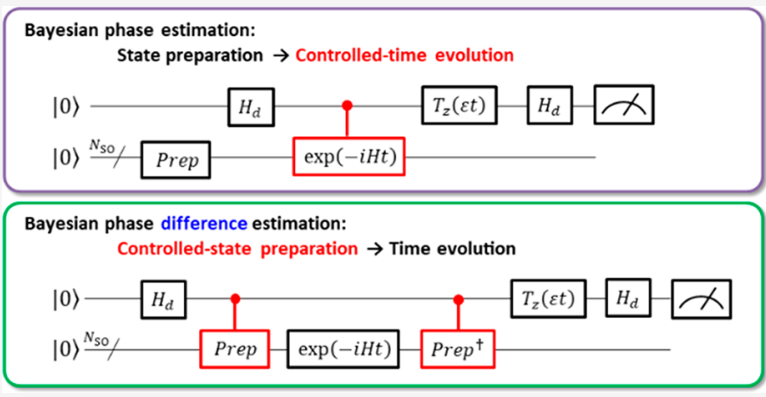

A ccurately solving a Schrödinger equation is one of the ultimate goals in chemistry and physics, and it opens the way toward predictive quantum chemistry. It allows us not only to deeply understand nature but also to design new materials with novel functionalities from the first principle's point of view, bringing a paradigm shift in scientific research and development. The full configuration interaction (full-CI) method gives numerically the best possible wave function for the space spanned by a given basis set, providing us the benchmarking results to which other approximate approaches can refer from the methodological point of view, and it is a practical goal of quantum chemical calculations. However, the computational cost of full-CI calculations grows exponentially against the increase of the basis set and of the number electrons relevant to the system size under study, and it can afford to deal with only small molecules; ${ }^{1,2}$ also it has been noted that putatively with the exception of strongly correlated systems and materials, there is no actual need to carry out fullCI calculations for chemical subjects of practical interest.

The appearance of quantum computers can change this scenario. As Feynman pointed out, quantum mechanical systems can be efficiently simulated by using a computer built of quantum mechanical elements that obey quantum mechanical laws. ${ }^{3}$ Indeed, Aspuru-Guzik and co-workers in 2005 proposed a quantum algorithm for full-CI calculations by utilizing a quantum phase estimation (QPE) algorithm, ${ }^{4}$ and proof-of-principle experiments of the full-CI/STO-3G of $\mathrm{H}_{2}$ by using photonic ${ }^{5}$ and $\mathrm{NMR}^{6}$ quantum computers were reported in 2010. Although the quantum circuit for QPE-based full-CI calculations is so deep that only small molecules like $\mathrm{H}_{2}$ and $\mathrm{HeH}^{+}$can be handled by the noisy intermediate-scale quantum devices currently available, ${ }^{5-8}$ it is believed to be a powerful tool when fault-tolerant quantum computing is realized, because an exponential speedup against classical algorithms is guaranteed.

Various theoretical attempts to improve the QPE-based methods have been reported so far, for example, improvement of computational space and Toffoli gate complexities using qubitization, $^{9-11}$ truncated Taylor series, ${ }^{12,13}$ linear combination of unitary $^{14}$ and tensor hypercontraction, ${ }^{15}$ quantum circuit optimizations, ${ }^{16}$ construction of approximated wave functions beyond Hartree-Fock (HF) level, ${ }^{17-19}$ and so on. Here, we focus on the modification of the QPE algorithm itself. In the QPE-based methods, time evolution of a wave function is simulated conditionally on an ancillary qubit, and the phase shift caused by the time evolution is read out by inverse quantum Fourier transformation ${ }^{20-22}$ or Bayesian inference. $^{23,24}$ The controlled time evolution simulation requires many controlled $R_{z}$ gates, whose fidelity is often lower than the single qubit $R_{z}$ gates used for unconditional time evolutions. The presence of many controlled $R_{z}$ gates also hinders parallel execution of quantum gates and compression of quantum circuits. Here, we take advantage of the Bayesian phase difference estimation (BPDE), recently proposed by us, ${ }^{25}$ to perform full-CI calculations without controlled time evolution operations. The BPDE algorithm allows us to directly compute the energy difference of two electronic states by conditional preparation of the quantum superposition of the two electronic states and following an unconditional time evolution. In

Received: September 30, 2021

Accepted: November 4, 2021

Published: November 8, 2021 
applying the BPDE algorithm to full-CI calculations, we construct the quantum superposition of vacuum and target electronic states. In other words, we compute the full-CI energy of an $\mathrm{N}$-electron system as the $\mathrm{N}$-electron ionization energy. To execute quantum chemical calculations on quantum computers, Fermionic wave functions should be mapped onto qubits by utilizing Fermion-qubit transformation techniques such as Jordan-Wigner transformation (JWT), ${ }^{4}$ BravyiKitaev transformation $(\mathrm{BKT}){ }^{26}$ and parity basis $(\mathrm{PB}){ }^{26}$ Importantly, if the JWT, BKT, or $\mathrm{PB}$ is adopted for the transformation, the quantum state initialized to the $|00 \cdots 0\rangle$ corresponds to the vacuum state having no electron. Note that the time evolution of the vacuum state under an electronic Hamiltonian causes no effects including that on the phase shift.

The quantum circuits for the full-CI calculations with Bayesian phase estimation (BPE) $)^{23}$ and $\mathrm{BPDE}^{25}$ are illustrated in Figure 1a,b, respectively. The definition of quantum gates is

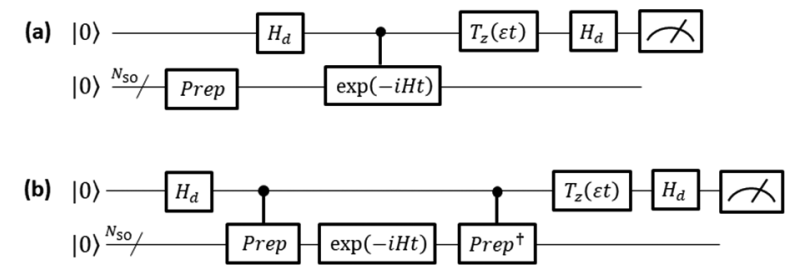

Figure 1. Quantum circuits for the full-CI calculations using (a) Bayesian phase estimation and (b) Bayesian phase difference estimation algorithms.

given in Supporting Information. The Prep gate in Figure 1 generates an approximated wave function of the target electronic state defined in eq 1 from the quantum state initialized to the $|00 \cdots 0\rangle$. The probability to obtain the $|0\rangle$ state in the measurement of the first qubit, $P(0)$, is calculated as in eq 2 .

$$
\begin{aligned}
& |\Phi\rangle=\sum_{j} c_{j}\left|\Psi_{j}\right\rangle \\
& P(0)=\left[1+\sum_{j}\left|c_{j}\right|^{2} \cos \left\{\left(E_{j}-\varepsilon\right) t\right\}\right] / 2
\end{aligned}
$$

Here, $\left|\Psi_{j}\right\rangle$ represents the $j$ th eigenfunction of the Hamiltonian, and $c_{j}$ is the corresponding expansion coefficient. In an ideal case, if $|\Phi\rangle$ is the pth eigenfunction of the Hamiltonian, the probability $P(0)$ becomes maximum when $\varepsilon=E_{p}$. Even if $|\Phi\rangle$ is described by a linear combination of many eigenfunctions, $P(0)$ has the upward peak at $\varepsilon \approx E_{p}$, as long as the expansion coefficient of the target electronic state, $c_{p}$, is sufficiently large. In $\mathrm{BPE}$, the phase rotation angle $\varepsilon$ is optimized by Bayesian inference to maximize $P(0)$. The controlled time evolution is used to extract the phase difference between the wave functions before and after the time evolution.

By contrast, in the BPDE-based implementation given in Figure $1 b$, the controlled operations appear in the state preparation and inverse state preparation, instead of the time evolution. Again, $P(0)$ is calculated as in eq 2, and thus the full$\mathrm{CI}$ energy can be obtained by optimizing the phase rotation angle $\varepsilon$ giving maximum $P(0)$. The BPDE-based implementation is more preferable than the conventional BPE if the quantum circuit for the Prep gate is shallower than that for the time evolution operation.
The Prep gate should have the ability to prepare the wave function having sufficiently large overlap with the target electronic state. For typical closed shell singlet molecules around their equilibrium geometry, the HF wave function, $\left|\Psi_{\mathrm{HF}}\right\rangle$, is generally a good approximation of the ground state wave function. In the JWT, $\left|\Psi_{\mathrm{HF}}\right\rangle$ can be prepared by $N_{\mathrm{e}}$ Pauli$X$ gates, where $N_{e}$ is the number of electrons. One can also prepare a spin symmetry-adapted configuration state function $(\mathrm{CSF})^{17,18}$ or a multiconfigurational wave function ${ }^{19}$ with shallow quantum circuits. The quantum circuit for the Prep gate is usually very short, and thus the number of two-qubit gates can be drastically reduced by adopting the BPDE-based approach.

To demonstrate the efficiency of the BPDE-based full-CI calculations, we developed a numerical quantum circuit simulation program using Python with OpenFermion ${ }^{27}$ and $\mathrm{Cirq}^{28}$ libraries. Detailed computational conditions are given in Supporting Information.

We focused on the potential energy curves of four valence electronic states $\left(S_{0}, S_{1}, S_{2}\right.$, and $\left.T_{1}\right)$ of the $H_{2}$ molecule with the 6-31G basis set. The wave functions of these electronic states can be well approximated by using eqs 3-6.

$$
\begin{aligned}
& \left|\Phi\left(\mathrm{S}_{0}\right)\right\rangle=\sqrt{1-y / 2}|2000\rangle-\sqrt{y / 2}|0200\rangle \\
& \left|\Phi\left(\mathrm{S}_{1}\right)\right\rangle=(|\alpha \beta 00\rangle-|\beta \alpha 00\rangle) / \sqrt{2} \\
& \left|\Phi\left(\mathrm{S}_{2}\right)\right\rangle=\sqrt{y / 2}|2000\rangle+\sqrt{1-y / 2}|0200\rangle \\
& \left|\Phi\left(\mathrm{T}_{1}\right)\right\rangle=|\alpha \alpha 00\rangle
\end{aligned}
$$

Here, $2, \alpha, \beta$, and 0 stand for doubly occupied, singly occupied by a spin- $\alpha$ electron, singly occupied by a spin- $\beta$ electron, and unoccupied molecular orbitals, respectively. $y$ in eqs 3 and 5 is a diradical character, which denotes a measure of the open shell electronic configuration. ${ }^{19,29,30}$ It can be calculated from the occupation number of the lowest unoccupied natural orbital $\left(n_{\text {LUNO }}\right)$ constructed from the spin-projected brokensymmetry (BS)-UHF wave functions, using eq $7 .^{29}$

$$
y=1-\frac{2\left(1-n_{\mathrm{LUNO}}\right)}{1+\left(1-n_{\mathrm{LUNO}}\right)^{2}}
$$

The quantum circuits of the controlled Prep gates are illustrated in Figure 2. The rotational angle $\theta$ in Figure 2a should be $-2 \operatorname{acos}(\sqrt{1-y / 2})$ and $2 \operatorname{acos}(\sqrt{y / 2})$ for the $S_{0}$ and $S_{2}$ states, respectively. The results of the BPDE quantum circuit simulations are plotted in Figure $3 \mathrm{a}$, and the deviations from the full-CI energy are given in Figure $3 b-e$. All the BPDE simulations converged after 4-5 Bayesian optimization cycles, and the length of total evolution time is about $180-280$ atomic units. The numerical simulations revealed that BPDE reproduces the full-CI energy within $3 \mathrm{kcal} \mathrm{mol}^{-1}$ of errors with ca. $0.05 \mathrm{kcal} \mathrm{mol}^{-1}$ of standard deviations. However, the plots in Figure $3 \mathrm{~b}-\mathrm{e}$ indicate the presence of somewhat systematic errors. The deviations mainly originate from Trotter decomposition errors, and they can be reduced by adopting shorter time length for the single Trotter slice. Current simulations use the time for a single Trotter slice $t / N=0.5$ atomic unit. By adopting five times finer Trotter decomposition, the $\triangle E(\mathrm{BPDE}-$ full-CI) values become less than 0.2 $\mathrm{kcal} \mathrm{mol}^{-1}$ for all the states and geometries (see Figure S7 in Supporting Information). Note that the computational cost is inversely proportional to the Trotter time steps $t / N$. We also 

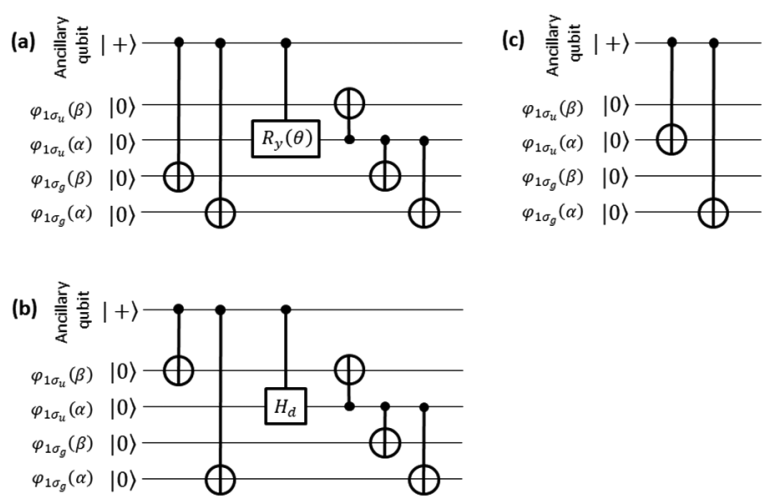

Figure 2. Quantum circuits of the controlled-Prep gates used for the full-CI calculations of the (a) $S_{0}$ and $S_{2}$, (b) $S_{1}$, and (c) $T_{1}$ states. The rotational angle $\theta$ is $-2 \operatorname{acos}(\sqrt{1-y / 2})$ and $2 \operatorname{acos}(\sqrt{y / 2})$ for the $S_{0}$ and $S_{2}$ states, respectively. Qubits storing the occupation number of $\varphi_{2 \sigma_{\mathrm{g}}}$ and $\varphi_{2 \sigma_{\mathrm{u}}}$ orbitals are omitted for clarity.
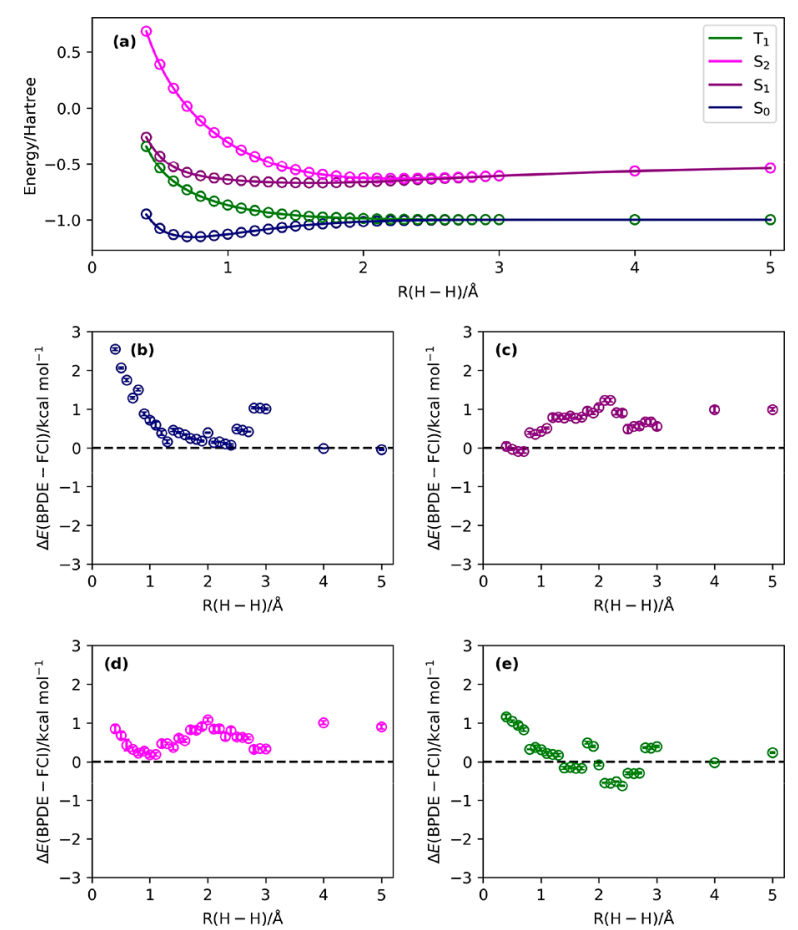

Figure 3. Numerical quantum circuit simulation results of the potential energy curves of the $\mathrm{H}_{2}$ molecule. (a) Total energies. Solid lines denote the full-CI energies, and open circles represent the BPDE results. (b-e) Energy deviations from the full-CI values in the (b) $S_{0}$, (c) $S_{1}$, (d) $S_{2}$, and (e) $T_{1}$ states. Error bars denote the standard deviations.

examined noisy quantum circuit simulations of the $S_{0}$ state of the $\mathrm{H}_{2}$ molecule with Pauli error channels. The noisy quantum circuit simulations gave larger standard deviations $(0.15 \mathrm{kcal}$ $\mathrm{mol}^{-1}$ on average), but the $\Delta E$ values are almost the same between the noiseless and noisy simulations (Figure S8 in Supporting Information), exemplifying the robustness of the BPDE algorithm.

Note that the number of Bayesian optimization cycles depends on the total energy and thus the system size, although it becomes less dependent on the system size for the direct calculation of energy gaps using the BPDE algorithm. ${ }^{25}$ Therefore, the two-step approach to calculate the total energy of the electronic ground state using the BPDE-based method described here and subsequently perform the direct calculation of the excitation energies with the BPDE algorithm can be more preferable to obtain the potential energy curves of excited states from the viewpoint of computational cost reduction.

We also applied the BPDE-based method to the energy diagram of the cis-trans-iso isomerization reaction of the diazene $\left(\mathrm{N}_{2} \mathrm{H}_{2}\right)$ molecule at the CAS-CI $(10 \mathrm{e}, 8 \mathrm{o}) / 6-311 \mathrm{G}$ $(\mathrm{d}, \mathrm{p})$ level. The two major reaction pathways of the cis-trans isomerization, namely, in-plane inversion and out-of-plane torsion, have been well investigated. ${ }^{31-34}$ Sophisticated quantum chemical calculations predicted that the transition state of the in-plane inversion (TS1 in Figure 4) has slightly

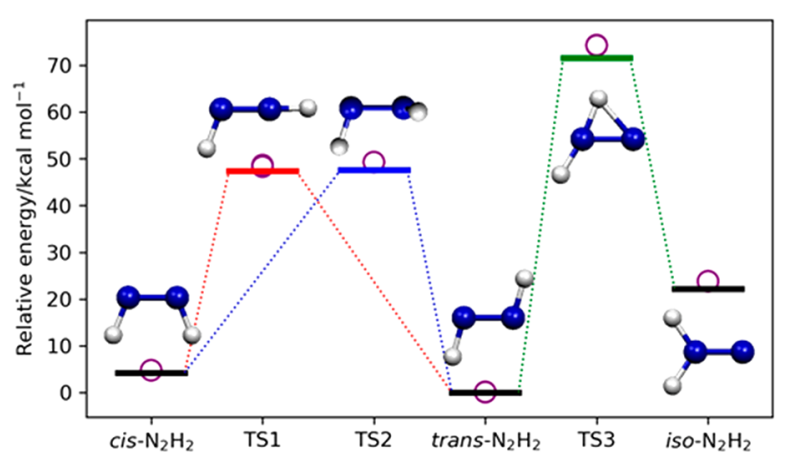

Figure 4. Potential energy profiles of the cis-trans-iso isomerization reaction of diazene, $\mathrm{N}_{2} \mathrm{H}_{2}$. Solid lines denote the CAS-CI energies, and purple circles represent the numerical simulation results.

lower energy than that of the out-of-plane torsion (TS2). ${ }^{32-34}$ From the viewpoint of transition energies, the two reaction pathways are competitive or the in-plane inversion is slightly favorable, but classical trajectory simulations revealed that the in-plane inversion is forbidden because of a centrifugal barrier, and the out-of-plane torsion is only the pathway of the isomerization reaction. ${ }^{34-36}$ Potential energy curves of these two reaction pathways were investigated at the HF level using variational quantum eigensolver $(\mathrm{VQE})^{37}$ with a superconducting quantum processor. ${ }^{38}$ However, it should be emphasized that in TS2 the $\mathrm{N}=\mathrm{N} \pi$ bond is broken and it exhibits a strong diradical character. The wave function of the lowest electronic state of TS2 cannot be well approximated at the HF level, and the post-HF calculation is essential to describe its electronic structure correctly. Numerical VQE simulations for low-lying electronic states and transition amplitudes in the cis-trans isomerization reaction pathway were also reported. ${ }^{39}$

The diradical character $y$ 's were calculated to be 0.0233 , $0.0373,0.9950$, and 0.1665 for iso- $\mathrm{N}_{2} \mathrm{H}_{2}$, TS1, TS2, and TS3, respectively. The BS-UHF calculations converged to the closed shell RHF state in the cis- and trans-isomers. In the simulations for the cis-, trans-, and iso-species, we used the controlled Prep gate that generates the RHF wave function. For the calculations of TS1, TS2, and TS3, we applied a quantum circuit similar to that in Figure $2 \mathrm{a}$ with $\theta=-2 \operatorname{acos}(\sqrt{1-y / 2})$ to prepare the two-configurational wave function. The results of the numerical simulations and the CAS-CI energies are plotted in Figure 4. The Bayesian optimization converged after 16 or 17 iterations. The energy differences between the BPDE simulations and the CAS-CI are 
$0.536,0.124,1.588,1.058,1.703$, and $2.680 \mathrm{kcal} \mathrm{mol}^{-1}$ for cis$\mathrm{N}_{2} \mathrm{H}_{2}$, trans $-\mathrm{N}_{2} \mathrm{H}_{2}$, iso $-\mathrm{N}_{2} \mathrm{H}_{2}$, TS1, TS2, and TS3, respectively, and the standard deviation is $0.05 \mathrm{kcal} \mathrm{mol}^{-1}$ on average. If we used the finer Trotter time steps, $t / N=0.1$ atomic unit, the $\Delta E$ (BPDE - full-CI) became 0.187, 0.013, 0.093, 0.160, 0.118, and $1.983 \mathrm{kcal} \mathrm{mol}^{-1}$ for cis $-\mathrm{N}_{2} \mathrm{H}_{2}$, trans $-\mathrm{N}_{2} \mathrm{H}_{2}$, iso $-\mathrm{N}_{2} \mathrm{H}_{2}, \mathrm{TS} 1$, TS2, and TS3, respectively. Only TS3 exhibits a large error in the simulations with the finer Trotter decompositions. This trend can be explained by the quality of the approximated wave function $|\Phi\rangle$. The square overlaps $\left|\left\langle\Phi \mid \Psi_{\text {CAS-CI }}\right\rangle\right|^{2}$ were calculated to be $0.9466,0.9499,0.9655,0.9435,0.9796$, and 0.8753 for cis $-\mathrm{N}_{2} \mathrm{H}_{2}$, trans $-\mathrm{N}_{2} \mathrm{H}_{2}$, iso- $\mathrm{N}_{2} \mathrm{H}_{2}$, TS1, TS2, and TS3, respectively. In TS3, contributions from electronic states other than the target state are not negligible, which shifts the $\varepsilon$ value giving maximum $P(0)$ in eq 2 from the value of $E_{0}$. Replacing the controlled Prep gate by more sophisticated wave function preparation techniques such as adiabatic state preparation (ASP) $)^{6,40}$ is necessary to acquire the energy of TS3 accurately. For such applications, the ASP should start from the quantum superposition of the vacuum state and the eigenfunction of a starting Hamiltonian as in eq 8 and execute normal (unconditioned) ASP.

$$
\left|\Phi_{\mathrm{ASP} ; \mathrm{Ini}}\right\rangle=\frac{1}{\sqrt{2}}\left(|0\rangle \otimes|\mathrm{vac}\rangle+|1\rangle \otimes\left|\Psi_{0 ; \text { Ini }}\right\rangle\right)
$$

In summary, we have examined a new quantum algorithm for full-CI calculations based on the BPDE algorithm. The proposed quantum algorithm is free from controlled-time evolution, making parallelization of quantum gates and implementation on real quantum devices easier. The proposed algorithm is easily connectable to ASP. Our algorithm could pave the way for more practical full-CI calculations on faulttolerant quantum computers.

\section{ASSOCIATED CONTENT}

\section{SI Supporting Information}

The Supporting Information is available free of charge at https://pubs.acs.org/doi/10.1021/acs.jpclett.1c03214.

Definitions of quantum gates, computational conditions for quantum chemical calculations and numerical quantum circuit simulations, Trotter decomposition error analysis, and quantum circuit simulations with Pauli error channels (PDF)

\section{AUTHOR INFORMATION}

\section{Corresponding Authors}

Kenji Sugisaki - Department of Chemistry and Molecular Materials Science, Graduate School of Science, Osaka City University, Osaka 558-8585, Japan; JST PRESTO, Kawaguchi, Saitama 332-0012, Japan; Centre for Quantum Engineering, Research and Education (CQuERE), TCG Centres for Research and Education in Science and Technology (TCG CREST), Kolkata 700091, India; ○ orcid.org/0000-0002-1950-5725; Email: sugisaki@ osaka-cu.ac.jp

Kazunobu Sato - Department of Chemistry and Molecular Materials Science, Graduate School of Science, Osaka City University, Osaka 558-8585, Japan; 이이.org/00000003-1274-7470; Email: sato@sci.osaka-cu.ac.jp

Takeji Takui - Department of Chemistry and Molecular Materials Science, Graduate School of Science, Osaka City University, Osaka 558-8585, Japan; Research Support
Department, University Research Administrator Center, University Administration Division, Osaka City University, Osaka 558-8585, Japan; (1) orcid.org/0000-0001-62385215; Phone: +81-6-6605-2605; Email: takui@sci.osakacu.ac.jp

\section{Authors}

Chikako Sakai - Department of Chemistry and Molecular Materials Science, Graduate School of Science, Osaka City University, Osaka 558-8585, Japan

Kazuo Toyota - Department of Chemistry and Molecular Materials Science, Graduate School of Science, Osaka City University, Osaka 558-8585, Japan

Daisuke Shiomi - Department of Chemistry and Molecular Materials Science, Graduate School of Science, Osaka City University, Osaka 558-8585, Japan; (1) orcid.org/00000002-7135-547X

Complete contact information is available at: https://pubs.acs.org/10.1021/acs.jpclett.1c03214

\section{Notes}

The authors declare no competing financial interest.

\section{ACKNOWLEDGMENTS}

This work was supported by JST PRESTO "Quantum Software" project (Grant No. JPMJPR1914), Japan, and KAKENHI Scientific Research C (Grant Nos. 18K03465 and 21K03407) from JSPS, Japan. This work was also supported by AOARD Scientific Project on "Molecular Spins for Quantum Technologies” (Grant FA2386-17-1-4040, 4041), USA.

\section{REFERENCES}

(1) Rossi, E.; Bendazzoli, G. L.; Evangelisti, S.; Maynau, D. A fullconfiguration benchmark for the $\mathrm{N}_{2}$ molecule. Chem. Phys. Lett. 1999, $310,530-536$.

(2) Gan, Z.; Grant, D. J.; Harrison, R. J.; Dixon, D. A. The lowest energy states of the group-IIIA-group-VA heteronuclear diatomics: $\mathrm{BN}, \mathrm{BP}, \mathrm{AlN}$, and AlP from full configuration interaction calculations. J. Chem. Phys. 2006, 125, 124311.

(3) Feynman, R. P. Simulating physics with computers. Int. J. Theor. Phys. 1982, 21, 467-488.

(4) Aspuru-Guzik, A.; Dutoi, A. D.; Love, P. J.; Head-Gordon, M. Simulated quantum computation of molecular energies. Science 2005, 309, 1704-1707.

(5) Lanyon, B. P.; Whitfield, J. D.; Gillett, G. G.; Goggin, M. E.; Almeida, M. P.; Kassal, I.; Biamonte, J. D.; Mohseni, M.; Powell, B. J.; Barbieri, M.; Aspuru-Guzik, A.; White, A. G. Towards quantum chemistry on a quantum computer. Nat. Chem. 2010, 2, 106-111.

(6) Du, J.; Xu, N.; Peng, X.; Wang, P.; Wu, S.; Lu, D. NMR implementation of a molecular hydrogen quantum simulation with adiabatic state preparation. Phys. Rev. Lett. 2010, 104, 030502.

(7) Wang, Y.; Dolde, F.; Biamonte, J.; Babbush, R.; Bergholm, V.; Yang, S.; Jakobi, I.; Neumann, P.; Aspuru-Guzik, A.; Whitfield, J. D.; Wrachtrup, J. Quantum simulation of helium hydride cation in a solid-state spin register. ACS Nano 2015, 9, 7769-7774.

(8) O'Malley, P. J. J.; et al. Scalable quantum simulation of molecular energies. Phys. Rev. X 2016, 6, 031007.

(9) Babbush, R.; Gidney, C.; Berry, D. W.; Wiebe, N.; McClean, J.; Paler, A.; Fowler, A.; Neven, H. Encoding electronic spectra in quantum circuits with linear $\mathrm{T}$ complexity. Phys. Rev. $X$ 2018, 8, 041015 .

(10) Berry, D. W.; Gidney, C.; Motta, M.; McClean, J. R.; Babbush, R. Qubitization of arbitrary basis quantum chemistry leveraging sparsity and low rank factorization. Quantum 2019, 3, 208. 
(11) von Burg, V.; Low, G. H.; Häner, T.; Steiger, D. S.; Reiher, M.; Roetteler, M.; Troyer, M. Quantum computing enhanced computational catalysis. Phys. Rev. Res. 2021, 3, 033055.

(12) Babbush, R.; Berry, D. W.; Kivlichan, I. D.; Wei, A. Y.; Love, P. J.; Aspuru-Guzik, A. Exponentially more precise quantum simulation of fermions in second quantization. New J. Phys. 2016, 18, 033032.

(13) Babbush, R.; Berry, D. W.; Sanders, Y. R.; Kivlichan, I. D.; Scherer, A.; Wei, A. Y.; Love, P. J.; Aspuru-Guzik, A. Exponentially more precise quantum simulation of fermions in the configuration interaction representation. Quantum Sci. Technol. 2018, 3, 015006.

(14) Childs, A. M.; Wiebe, N. Hamiltonian simulation using linear combinations of unitary operations. Quantum Inf. Comput. 2012, 12, 901-924.

(15) Lee, J.; Berry, D. W.; Gidney, C.; Huggins, W. J.; McClean, J. R.; Wiebe, N.; Babbush, R. Even more efficient quantum computations of chemistry through tensor hypercontraction. PRX Quantum 2021, 2, 030305.

(16) Hastings, M. B.; Wecker, D.; Bauer, B.; Troyer, M. Improving quantum algorithms for quantum chemistry. Quantum Inf. Comput. $2015,15,1-21$

(17) Sugisaki, K.; Yamamoto, S.; Nakazawa, S.; Toyota, K.; Sato, K.; Shiomi, D.; Takui, T. Quantum chemistry on quantum computers: A polynomial-time quantum algorithm for constructing the wave functions of open-shell molecules. J. Phys. Chem. A 2016, 120, 6459-6466.

(18) Sugisaki, K.; Yamamoto, S.; Nakazawa, S.; Toyota, K.; Sato, K.; Shiomi, D.; Takui, T. Open shell electronic state calculations on quantum computers: A quantum circuit for the preparation of configuration state functions based on Serber construction. Chem. Phys. Lett. 2019, 737, 100002.

(19) Sugisaki, K.; Nakazawa, S.; Toyota, K.; Sato, K.; Shiomi, D.; Takui, T. Quantum chemistry on quantum computers: A method for preparation of multiconfigurational wave functions on quantum computers without post-Hartree-Fock calculations. ACS Cent. Sci. 2019, 5, 167-175.

(20) Abrams, D. S.; Lloyd, S. Quantum algorithm providing exponential speed increase for finding eigenvalues and eigenvectors. Phys. Rev. Lett. 1999, 83, 5162-5165.

(21) Dobšíček, M.; Johansson, G.; Shumeiko, V.; Wendin, G. Arbitrary accuracy iterative quantum phase estimation algorithm using a single ancillary qubit: A two-qubit benchmark. Phys. Rev. A: At., Mol., Opt. Phys. 2007, 76, 030306.

(22) Nielsen, M. A.; Chuang, I. L. Quantum Computation and Quantum Information, 10th anniversary ed.; Cambridge University Press: Cambridge, UK, 2010.

(23) Wiebe, N.; Granade, C. Efficient Bayesian phase estimation. Phys. Rev. Lett. 2016, 117, 010503.

(24) Paesani, S.; Gentile, A. A.; Santagati, R.; Wang, J.; Wiebe, N.; Tew, D. P.; O’Brien, J. L.; Thompson, M. G. Experimental Bayesian quantum phase estimation on a silicon photonic chip. Phys. Rev. Lett. 2017, 118, 100503.

(25) Sugisaki, K.; Sakai, C.; Toyota, K.; Sato, K.; Shiomi, D.; Takui, $\mathrm{T}$. Bayesian phase difference estimation: a general quantum algorithm for the direct calculation of energy gaps. Phys. Chem. Chem. Phys. 2021, 23, 20152-20162.

(26) Seeley, J. T.; Richard, M. J.; Love, P. J. The Bravyi-Kitaev transformation for quantum computation of electronic structure. $J$. Chem. Phys. 2012, 137, 224109.

(27) McClean, J. R.; Rubin, N. C.; Sung, K. J.; Kivlichan, I. D.; Bonet-Monroig, X.; Cao, Y.; Dai, C.; Fried, E. S.; Gidney, C.; Gimby, B.; et al. OpenFermion: the electronic structure package for quantum computers. Quantum Sci. Technol. 2020, 5, 034014.

(28) Quantum AI team and collaborators. quantumlib/Cirq. Cirq, version v0.10.0. Zenodo, March 5, 2021, DOI: 10.5281/zenodo.4586899.

(29) Yamaguchi, K. In Self-consistent field: Theory and applications; Carbo, R., Klobukowski, M., Eds; Elsevier: Amsterdam, 1990; pp 727-828.
(30) Döhnert, D.; Koutecky, J. Occupation numbers of natural orbitals as a criterion for biradical character. Different kinds of biradicals. J. Am. Chem. Soc. 1980, 102, 1789-1796.

(31) Jensen, H. J. A.; Jørgensen, P.; Helgaker, T. The ground-state potential energy surface of diazene. J. Am. Chem. Soc. 1987, 109, 2895-2901.

(32) Biczysko, M.; Poveda, L. A.; Varandas, A. J. C. Accurate MRCI study of ground-state $\mathrm{N}_{2} \mathrm{H}_{2}$ potential energy surface. Chem. Phys. Lett. 2006, 424, 46-53.

(33) Chaudhuri, R. K.; Freed, K. F.; Chattopadhyay, S.; Mahapatra, U. S. Potential energy curve for isomerization of $\mathrm{N}_{2} \mathrm{H}_{2}$ and $\mathrm{C}_{2} \mathrm{H}_{4}$ using the improved virtual orbital multireference Møller-Plesset perturbation theory. J. Chem. Phys. 2008, 128, 144304.

(34) Sindhu, A.; Pradhan, R.; Lourderaj, U.; Paranjothy, M. Theoretical investigation of the isomerization pathways of diazenes: torsion vs. inversion. Phys. Chem. Chem. Phys. 2019, 21, 1567815685 .

(35) Spears, J. G., Jr.; Hutchinson, J. S. Classical dynamics of transdiimide: Intramolecular vibrational relaxation involving an active torsion. J. Chem. Phys. 1988, 88, 240-249.

(36) Spears, J. G., Jr.; Hutchinson, J. S. Classical dynamics of an internal Coriolis-induced isomerization of trans-diimine. J. Chem. Phys. 1988, 88, 250-259.

(37) Peruzzo, A.; McClean, J.; Shadbolt, P.; Yung, M.-H.; Zhou, X.Q.; Love, P. J.; Aspuru-Guzik, A.; O’Brien, J. L. A variational eigenvalue solver on a photonic quantum processor. Nat. Commun. 2014, 5, 4213.

(38) Arute, F.; et al. Hartree-Fock on a superconducting qubit quantum computer. Science 2020, 369, 1084-1089.

(39) Ibe, Y.; Nakagawa, Y. O.; Earnest, N.; Yamamoto, T.; Mitarai, K.; Gao, Q.; Kobayashi, T. Calculating transition amplitudes by variational quantum deflation. arXiv 2020, arXiv:2002.11724, https:// arxiv.org/abs/2002.11724.

(40) Veis, L.; Pittner, J. Adiabatic state preparation study of methylene. J. Chem. Phys. 2014, 140, 214111. 